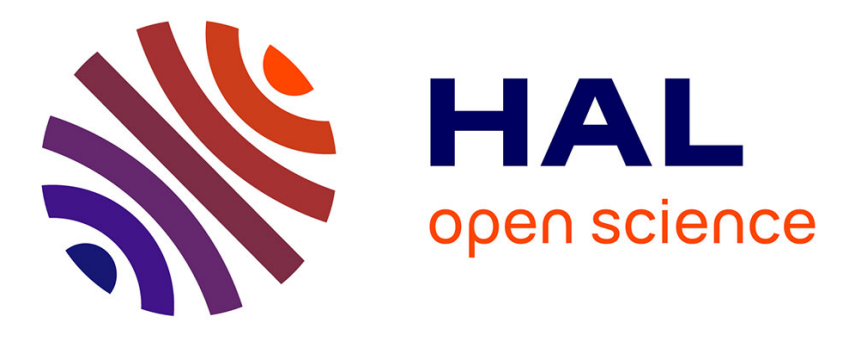

\title{
Etude de populations européennes de maïs pour quelques caractères d'intérêt agronomique
}

François Kaan, Sylvain Rautou, Antoine Panouillé, Armand Boyat, Michel

Tersac, Jacques Cognot, Michel Dupin, Jean Vivares, Marc Bedere, Bernard Fresnay, et al.

\section{To cite this version:}

François Kaan, Sylvain Rautou, Antoine Panouillé, Armand Boyat, Michel Tersac, et al.. Etude de populations européennes de maïs pour quelques caractères d'intérêt agronomique. Agronomie, 1982, 2 (6), pp.577-582. hal-00884420

\section{HAL Id: hal-00884420 https://hal.science/hal-00884420}

Submitted on 1 Jan 1982

HAL is a multi-disciplinary open access archive for the deposit and dissemination of scientific research documents, whether they are published or not. The documents may come from teaching and research institutions in France or abroad, or from public or private research centers.
L'archive ouverte pluridisciplinaire HAL, est destinée au dépôt et à la diffusion de documents scientifiques de niveau recherche, publiés ou non, émanant des établissements d'enseignement et de recherche français ou étrangers, des laboratoires publics ou privés. 


\title{
Etude de populations européennes de maïs pour quelques caractères d'intérêt agronomique
}

\author{
François KAAN, Sylvain RAUTOU, Antoine PANOUILlÉ $\left({ }^{*}\right)$, Armand BOYAT \& Michel TERSAC \\ avec la collaboration de Jacques COGNOT, Michel DUPIN $(*)$, Jean VIVARES $\left({ }^{*}\right)$, Marc BedERE $(*)$, \\ Bernard FRESNAY \& Christiane BORRIES \\ I.N.R.A., Station d'Amélioration des Plantes, 9, Place Viala, F 34060 Montpellier Cedex. \\ (*) I.N.R.A., Station Expérimentale du Maïs, Saint-Martin-de-Hinx, F 40390 Saint-Martin-de-Seignanx.
}

\begin{abstract}
RÉSUMÉ
En 1977, 175 populations locales de maïs originaires de Bulgarie, France, Hongrie, Roumanie, Tchécoslovaquie et Yougoslavie ont été étudiées dans 2 stations, pour 10 caractères : vigueur de départ, précocité de

Maïs,

Zea mays $L$,

Population,

Europe,

Sélection.

floraison femelle, protandrie, pourriture des tiges, verse à la récolte, nombre d'épis pour 100 pieds, santé de l'épi, teneur en protéine du grain, texture et couleur du grain.

Tous ces caractères montrent une variation significative selon le pays d'origine. Quinze associations significatives entre 2 caractères ont été trouvées. Elles peuvent être attribuées dans certains cas à des effets génétiques (par exemple pléiotropie), à des effets de sélection, à des effets multivariables (corrélations multiples), ou plus simplement à des effets d'échantillonnage.
\end{abstract}

\section{SUMMARY}

Maize,

Zea Mays $L$.,

Population,

Europe,

Breeding.

\section{A study of European maize populations for certain agronomic traits}

175 local maize populations from Bulgaria, Czechoslovakia, France, Hungary, Romania and Yugoslavia were grown in 1977 at 2 stations and observed for 10 traits : early vigour, silking date, protandrism, stalkrot, lodging at harvest, ear number, ear health, kernel protein content, kernel texture and colour. All characters showed significant variation in relation to the different countries of origin.

Fifteen significant associations between 2 traits were found. In some instances, these relations could be due to genetical effects (for example pleiotropy), breeding effects, multivariate effects (multiple correlations), and sampling effects.

\section{INTRODUCTION}

De nombreux travaux effectués sur des collections nationales ou internationales décrivent et classent les populations européennes de maïs (SANCHEZ-MONGE, 1962 et 1964 ; EDWARDS \& LENG, 1965 ; MONTEAGUdo, 1967 ; MURESAN \& Cosmin, 1967 ; Pavlicic \& TRIfunoviC, 1967 ; PAVliCIC, 1969 ; BRANDOLINI, 1970). Ils font clairement apparaître une ample variabilité morphologique et physiologique.

D'un point de vue agronomique, ces populations sont rarement cultivées en raison de leur faible valeur propre comparée à celle des meilleures combinaisons hybrides commerciales. Mis à part quelques cas exemplaires, ces populations n'ont guère été exploitées par les sélectionneurs afin d'en tirer directement des lignées dites de « $1^{\text {er }}$ cycle » utilisables dans des combinaisons hybrides.

Une exploitation indirecte de ces populations peut néanmoins être entreprise en relation avec le nécessaire élargissement de la base de variabilité génétique, grâce aux méthodes actuelles de sélection récurrente des populations qui permettent une mise en œuvre à moyen et à long terme.
Les observations que nous avons réalisées en 1977 sur un échantillonnage important étaient destinées à apprécier la variabilité entre populations pour 6 caractères d'intérêt économique (vigueur de départ, précocité de floraison femelle, pourriture des tiges, verse à la récolte, santé de l'épi, teneur en protéine du grain), 2 caractères physiologiques (protandrie, nombre d'épis pour 100 pieds), 2 caractères morphologiques (texture et couleur du grain).

Nous avons laissé de côté dans cette $1^{\text {re }}$ étude l'appréciation du rendement, caractère complexe, de faible héritabilité.

\section{MATÉRIEL ET MÉTHODES}

Cent soixante-quinze populations locales ont été étudiées à l'exclusion des synthétiques ou composites: 65 provenaient de Bulgarie, 41 de France, 19 de Hongrie, 32 de Roumanie, 2 de Tchécoslovaquie et 16 de Yougoslavie.

En 1977, 4 parcelles de 30 plantes de chaque population. ont été implantées : 2 parcelles à Montpellier (Hérault), 2 à 
Saint-Martin-de-Hinx (Landes). Dans chaque lieu, les parcelles étaient peuplées à 2 densités $(50000$ et 73500 plantes/ha). Les cultures ont été menées dans des conditions comparables à celles d'une production commerciale.

Dix caractères ont été étudiés :

1) vigueur de départ (VD) : appréciation visuelle de la masse foliaire de 0 à 10 au stade 4-5 feuilles, transformée en radians $\left(2 \operatorname{Arcs} \sin \sqrt{\frac{x}{10}}\right)$, sur 4 répétitions;

2) précocité de floraison femelle $(\mathrm{FF})$ : nombre de jours écoulés entre le semis et la date médiane d'apparition des soies, sur 4 répétitions;

3) protandrie (PA) : nombre de jours écoulés jusqu'à la floraison femelle diminué du nombre de jours jusqu'à la floraison mâle, sur 4 répétitions ;

4) pourriture des tiges (PT) : la notation a été faite à la maturité apparente de chaque population, environ $60 \mathrm{j}$ après la floraison, par pression manuelle, en pourcentage de plantes versées sur les 2 répétitions à St-Martin-de-Hinx, transformé en radians $\left(2 \operatorname{Arc} \sin \sqrt{\frac{x}{100}}\right)$;

5) verse à la récolte (VR): la notation a été faite à la maturité apparente de chaque population, par observation du pourcentage de plantes versées sur les 2 répétitions de St-Martin-de-Hinx, transformé en radians (2 Arc $\left.\sin \sqrt{\frac{x}{100}}\right)$;

6) nombre d'épis pour 100 pieds (NE): sur 3 répétitions ;

7) santé de l'épi (SE) : la notation a été faite à la maturité apparente de chaque population par notation visuelle de 0 (épis moisis) à 10 (épis sains) sur les 2 répétitions de StMartin-de-Hinx, transformée en radians $\left(2 \operatorname{Arcs} \sin \sqrt{\frac{x}{10}}\right)$;
8) Teneur en protéine du grain (PG) mesurée par spectrométrie de réflexion dans le proche infra-rouge sur un échantillon de grains produits la même année, en pourcentage ;

9) texture du grain : corné, corné à corné denté, corné denté, denté corné, denté, notée sur 3 répétitions. Pour simplifier l'interprétation, nous avons considéré seulement 2 classes : corné et «denté », la classe «denté » comprend toutes les catégories sauf corné ;

10) couleur de l'albumen du grain : blanc, jaune, orange, notée sur 3 répétitions. Pour simplifier l'interprétation, toutes les populations blanches ou hétérogènes pour la couleur blanche ont été classées «blanches», les autres « jaunes».

Pour tous les caractères quantitatifs sauf la teneur en protéine du grain, l'écart-type de l'erreur sur les moyennes des populations est tiré de l'analyse de variance de l'essai. Pour la teneur en protéine du grain, l'écart-type de l'erreur est estimé à partir d'un essai réalisé la même arınée dans des conditions comparables.

\section{RÉSULTATS}

Une variation significative est observée pour tous les caractères quantitatifs : vigueur de départ, précocité de floraison femelle, protandrie, pourriture des tiges, verse à la récolte, nombre d'épis pour 100 pieds, santé de l'épi, teneur en protéine du grain (tabl.1). On note également une variation significative pour les caractères qualitatifs (texture et couleur du grain) (tabl. 2).

\section{TABLEAU 1}

Moyennes, variances, erreurs et corrélations entre caractères quantitatifs.

Means, variances, errors and correlations between quantitative traits.

\begin{tabular}{|c|c|c|c|c|c|c|c|c|c|}
\hline & $\begin{array}{c}\text { Vigueur } \\
\text { de départ } \\
\text { (Early } \\
\text { vigour) } \\
\text { VD }\end{array}$ & $\begin{array}{l}\text { Précocité de } \\
\text { floraison } \\
\text { femelle } \\
\text { (Days to silk) } \\
\text { FF }\end{array}$ & $\begin{array}{c}\text { Protandrie } \\
\text { (Protandrism) } \\
\text { PA }\end{array}$ & $\begin{array}{c}\text { Pourriture } \\
\text { des tiges } \\
\text { (Stalkrot) } \\
\text { PT }\end{array}$ & $\begin{array}{c}\text { Verse à la } \\
\text { récolte } \\
\text { (Harvest } \\
\text { lodged) } \\
\text { VR }\end{array}$ & $\begin{array}{c}\text { Nombre d'épis } \\
\text { pour } \\
100 \text { pieds } \\
\text { (Ear number) } \\
\text { NE }\end{array}$ & $\begin{array}{c}\text { Santé de } \\
\text { l'épi } \\
\text { (Ear health) } \\
\text { SE }\end{array}$ & $\begin{array}{l}\text { Teneur en } \\
\text { protéine } \\
\text { du grain } \\
\text { (Kernel } \\
\text { protein } \\
\text { content) } \\
\text { PG }\end{array}$ & $\begin{array}{c}\text { Caractère } \\
\text { (Trait) } \\
\text { Sigle }\end{array}$ \\
\hline \multirow{3}{*}{ 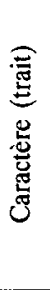 } & 1,83 & 84,82 & 2,73 & 1,62 & 1,51 & 96,71 & 2,18 & 12,04 & $\begin{array}{l}\text { moyenne générale } \\
(\text { mean })\end{array}$ \\
\hline & 0,19 & 5,14 & 1,16 & 0,47 & 0,37 & 9,06 & 0,22 & 1,06 & $\begin{array}{l}\text { écart type des moyennes } \\
\text { des populations } \\
\text { (mean S.E.) }\end{array}$ \\
\hline & 0,08 & 0,92 & 0,66 & 0,21 & 0,16 & 5,82 & 0,15 & 0,67 & $\begin{array}{l}\text { écart-type de } \\
\text { l'erreur (error S.E.) }\end{array}$ \\
\hline $\begin{array}{l}\text { VD } \\
\text { FF }\end{array}$ & & $-0,13$ & $\begin{array}{l}0,00 \\
+0,48^{* *}\end{array}$ & $\begin{array}{l}-0,14 \\
-0,28^{* *}\end{array}$ & $\begin{array}{l}0,00 \\
+0,22^{* *}\end{array}$ & $\begin{array}{l}+0,03 \\
-0,15^{*}\end{array}$ & $\begin{array}{l}+0,26^{* *} \\
-0,09\end{array}$ & $\begin{array}{l}-0,07 \\
-0,03\end{array}$ & \multirow{3}{*}{$\begin{array}{c}\text { Coefficient de } \\
\text { corrélation } \\
\text { (Correlation } \\
\text { coefficient) }\end{array}$} \\
\hline $\begin{array}{l}\text { PA } \\
\text { PT }\end{array}$ & & & & $-0,19^{*}$ & $\begin{array}{l}+0,08 \\
-0,06\end{array}$ & $\begin{array}{l}-0,31^{* *} \\
+0,15^{*}\end{array}$ & $\begin{array}{l}-0,09 \\
-0,42^{* *}\end{array}$ & $\begin{array}{l}+0,05 \\
+0,17^{*}\end{array}$ & \\
\hline $\begin{array}{l}\text { VR } \\
\text { NE } \\
\text { SE }\end{array}$ & & & & & & $-0,11$ & $\begin{array}{r}-0,07 \\
+0,14\end{array}$ & $\begin{array}{l}-0,01 \\
-0,08 \\
-0,23^{* *}\end{array}$ & \\
\hline
\end{tabular}


TABLEAU 2

Différences entre les pays d'origine pour les caractères qualitatifs. Differences between countries for qualitative traits.

\begin{tabular}{|c|c|c|c|c|c|c|c|c|c|c|}
\hline 胥焉 & $\stackrel{\square}{a}$ & 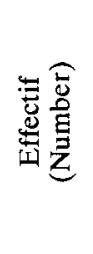 & 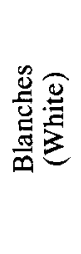 & 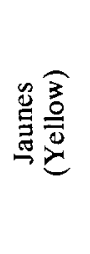 & 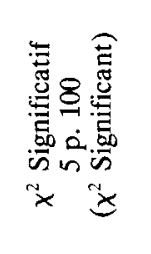 & 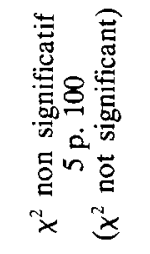 & 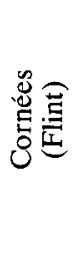 & 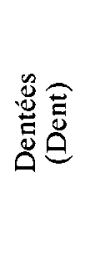 & 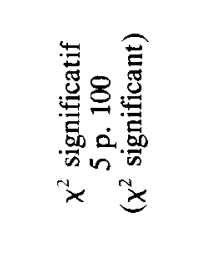 & 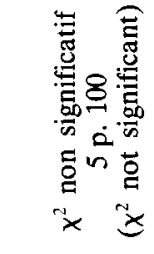 \\
\hline $\begin{array}{l}\text { Bulgarie } \\
\text { (Bulgaria) }\end{array}$ & BG & 65 & 10 & 55 & $F, Y U$ & $\mathrm{H}, \mathrm{R}$ & 37 & 28 & $F, Y U$ & $\mathrm{H}, \mathrm{R}$ \\
\hline France & $\mathrm{F}$ & 41 & 24 & 17 & $\mathrm{BG}, \mathrm{R}$ & $\mathrm{H}, \mathrm{YU}$ & 38 & 3 & $\mathrm{BG}, \mathrm{H}, \mathrm{R}, \mathrm{YU}$ & \\
\hline $\begin{array}{l}\text { Hongrie } \\
\text { (Hungary) }\end{array}$ & $\mathbf{H}$ & 19 & 5 & 14 & YU & $\mathrm{BG}, \mathrm{F}, \mathrm{R}$ & 9 & 10 & $F, R$ & $\mathrm{BG}, \mathrm{YU}$ \\
\hline $\begin{array}{l}\text { Roumanie } \\
\text { (Romania) }\end{array}$ & $\mathbf{R}$ & 32 & 4 & 28 & $\mathrm{~F}, \mathrm{YU}$ & $\mathrm{BG}, \mathrm{H}$ & 19 & 13 & $\mathrm{~F}, \mathrm{H}, \mathrm{YU}$ & BG \\
\hline $\begin{array}{l}\text { Tchécoslovaquie } \\
\text { (Czechoslovakia) }\end{array}$ & & 2 & 2 & 0 & & & 2 & 0 & & \\
\hline $\begin{array}{l}\text { Yougoslavie } \\
\text { (Yugoslavia) }\end{array}$ & YU & 16 & 16 & 0 & $\mathrm{BG}, \mathrm{H}, \mathrm{R}$ & $\mathrm{F}$ & 2 & 14 & $\mathrm{BG}, \mathrm{F}, \mathrm{R}$ & $\mathrm{H}$ \\
\hline Total & & 175 & 61 & 114 & & & 107 & 68 & & \\
\hline
\end{tabular}

TABLEAU 3

Variation des caractères quantitatifs en fonction du pays d'origine. Variation of quantitative traits in relation to the country of introduction.

\begin{tabular}{|c|c|c|c|c|c|c|c|c|c|}
\hline \multirow{3}{*}{$\begin{array}{c}\text { Pays } \\
\text { (Country) }\end{array}$} & \multirow{3}{*}{ 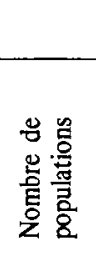 } & \multicolumn{8}{|c|}{ Caractère (trait) } \\
\hline & & $\begin{array}{l}\text { Vigueur } \\
\text { de départ } \\
\text { (Early } \\
\text { Vigour) }\end{array}$ & $\begin{array}{c}\text { Précocité } \\
\text { de floraison } \\
\text { femelle } \\
\text { (Days to silk) }\end{array}$ & $\begin{array}{c}\text { Protandrie } \\
\text { (Protandrism) }\end{array}$ & $\begin{array}{c}\text { Pourriture } \\
\text { des tiges } \\
\text { (Stalkrot) }\end{array}$ & $\begin{array}{l}\text { Verse à la } \\
\text { récolte } \\
\text { (Harvest } \\
\text { lodged) }\end{array}$ & $\begin{array}{c}\text { Nombre } \\
\text { d'épis pour } \\
100 \text { pieds } \\
\text { (Ear number) }\end{array}$ & $\begin{array}{c}\text { Santé } \\
\text { de l'épi } \\
\text { (Ear hcalth) }\end{array}$ & $\begin{array}{l}\text { Protéinc } \\
\text { du grain } \\
\text { (Kernel } \\
\text { protein }\end{array}$ \\
\hline & & VD & FF & $\mathrm{PA}$ & PT & VR & $\mathrm{NE}$ & SE & PG \\
\hline $\begin{array}{l}\text { Bulgarie } \\
\text { (Bulgaria) }\end{array}$ & 65 & $1,83 \mathrm{~b}$ & 84,04 b & $2,60 \mathrm{a}$ & $1,85 \mathrm{c}$ & $1,48 \mathrm{a}$ & 98,40 a & $2,13 \mathrm{c}$ & $12,44 \mathrm{a}$ \\
\hline France & 41 & $1,88 \mathrm{a}$ & $82,43 \mathrm{a}$ & $2,76 \mathrm{ab}$ & $1,39 \mathrm{a}$ & $1,49 \mathrm{a}$ & 96,54 a & $2,31 \mathrm{a}$ & $11,66 \mathrm{~cd}$ \\
\hline $\begin{array}{l}\text { Hongrie } \\
\text { (Hungary) }\end{array}$ & 19 & $1,84 a b$ & $87,34 \mathrm{~d}$ & $3,00 \mathrm{~b}$ & $1,46 \mathrm{ab}$ & $1,55 \mathrm{ab}$ & $90,89 \mathrm{~b}$ & $2,00 \mathrm{~d}$ & $12,03 \mathrm{bc}$ \\
\hline $\begin{array}{l}\text { Roumanie } \\
\text { (Romania) }\end{array}$ & 32 & $1,84 \mathrm{~b}$ & $86,03 \mathrm{c}$ & $2,60 \mathrm{a}$ & $1,56 \mathrm{~b}$ & $1,57 \mathrm{~b}$ & $99,06 \mathrm{a}$ & $2,20 \mathrm{~b}$ & $12,11 \mathrm{ab}$ \\
\hline $\begin{array}{l}\text { Yougoslavie } \\
\text { (Yugoslavia) }\end{array}$ & 16 & $1,79 \mathrm{~b}$ & $88,44 \mathrm{e}$ & $3,00 \mathrm{~b}$ & $1,60 \mathrm{~b}$ & $1,46 \mathrm{a}$ & 96,69 a & $2,15 b c$ & $11,31 \mathrm{~d}$ \\
\hline $\begin{array}{l}\text { Les moyenn } \\
\text { favorable. } \\
\text { Means with }\end{array}$ & ectées & $\begin{array}{l}\text { une lettro } \\
\text { s differ }\end{array}$ & $\begin{array}{l}\text { fférente sont } \\
\text { istically (Stud }\end{array}$ & $\begin{array}{l}\text { distinctes au } \\
\text { dent test, } 5 \%\end{array}$ & $\begin{array}{l}15 \mathrm{p} .10 \\
\text { it). } \mathrm{A} \mathrm{c}\end{array}$ & $\begin{array}{l}\text { de Stude } \\
\text { onds to } t\end{array}$ & $\begin{array}{l}\text { La lettre a } \\
\text { best level. }\end{array}$ & & iveau le \\
\hline
\end{tabular}




\section{A. Différences entre pays d'origine}

La signification de ces résultats est relativement difficile à dégager en raison du nombre variable de populations introduites de chaque pays. La Tchécoslovaquie est exclue de la récapitulation par pays pour les caractères quantitatifs (tabl. 3) car nous n'avons pu examiner que 2 populations originaires de ce pays.

Des différences significatives entre certains pays apparaissent pour tous les caractères quantitatifs (tabl. 3 ).

Pour les 2 caractères qualitatifs, des différences significatives entre certains pays apparaissent également (tabl. 2).

\section{B. Associations entre caractères}

Pour les 8 caractères quantitatifs, 11 corrélations entre 2 caractères apparaissent significatives au seuil 5 p. 100 , dont 7 au seuil 1 p. 100 (tabl. 1).

Pour éprouver les associations entre caractères quantitatifs et qualitatifs, les moyennes de chaque classe qualitative pour chaque caractère quantitatif ont été comparées (tabl. 4). Les 16 associations possibles ont été étudiées : 4 sont significatives au seuil 1 p. 100 dont 3 au seuil 1 p. 1000 . Il n'y a pas d'association significative entre les 2 caractères qualitatifs (texture et couleur du grain).

\section{DISCUSSION}

\section{A. Différences entre pays d'origine}

De tous les pays étudiés, la France est le seul qui jouisse d'un climat atlantique, relativement frais et humide. Il faut peut-être mettre ce facteur en relation avec le $1^{\text {er }}$ rang des populations françaises sur la vigueur de départ, la précocité de floraison femelle, la résistance à la pourriture des tiges, la santé de l'épi.

Contrairement à ce que l'on pourrait supposer, les populations hongroises étudiées sont plus tardives que les bulgares, pourtant plus méridionales. Cette adaptation douteuse des populations hongroises à nos conditions expérimentales est confirmée par leur faible nombre d'épis par plantes (90,9 épis pour 100 pieds seulement).

L'importante proportion de génotypes dentés chez le matériel hongrois étudié pourrait indiquer également une influence d'introductions nord-américaines relativement récentes ; il est toutefois possible que notre échantillonnage soit trop restrictif.

Les populations étudiées sont en prédominance cornées pures, sauf en Hongrie et en Yougoslavie.

Les habitudes alimentaires et d'élevage expliquent peutêtre que tous nos échantillons yougoslaves soient à grain blanc, ainsi que l'importance des populations blanches en France et en Hongrie, pays où le gavage des volailles constitue une ressource importante. La forte teneur en protéine des échantillons bulgares peut être mise en relation avec la texture nécessaire à la confection de la «mamaliga », recette nationale bulgare.

\section{B. Association entre caractères}

Cinq facteurs principaux peuvent expliquer les associations constatées entre 2 caractères (tabl. 1 et 4 ) :

1) la pléiotropie génétique, assimilable à une corrélation physiologique ;

2) le linkage chromosomique ;

3) la sélection;

\section{TABLEAU 4}

Relations entre caractères quantitatifs et qualitatifs.

Relations between quantitative and qualitative traits.

\begin{tabular}{|c|c|c|c|c|c|c|c|c|}
\hline & \multicolumn{8}{|c|}{ Caractèrc quantitatif (Quantitative trait) } \\
\hline & $\begin{array}{l}\text { Vigueur } \\
\text { de départ } \\
\text { (Early } \\
\text { vigour) }\end{array}$ & $\begin{array}{l}\text { Précocité } \\
\text { de floraison } \\
\text { femelle } \\
\text { (Days to silk) }\end{array}$ & $\begin{array}{l}\text { Protandrie } \\
\text { (Protandrism) }\end{array}$ & $\begin{array}{l}\text { Pourriture } \\
\text { des tiges } \\
\text { (Stalkrot) }\end{array}$ & $\begin{array}{l}\text { Verse à la } \\
\text { récolte } \\
\text { (Harvest } \\
\text { lodged) }\end{array}$ & $\begin{array}{c}\text { Nombre } \\
\text { d'épis pour } \\
100 \text { pieds } \\
\text { (Ear number) }\end{array}$ & $\begin{array}{c}\text { Santé } \\
\text { de l'épi } \\
\text { (Ear health) }\end{array}$ & $\begin{array}{l}\text { Protéine } \\
\text { du grain } \\
\text { (Kernel } \\
\text { protein } \\
\text { content) }\end{array}$ \\
\hline & VD & $\mathrm{FF}$ & $\mathrm{PA}$ & PT & VR & $\mathrm{NE}$ & SE & PG \\
\hline \multicolumn{9}{|l|}{$\begin{array}{l}\text { Caractère qualitatif du grain (qualita- } \\
\text { tive kernel trait) }\end{array}$} \\
\hline Corné (flint) 108 populations & 1,82 & 83,25 & 2,69 & 1,69 & 1,50 & 96,81 & 2,16 & 12,15 \\
\hline Denté (dent) 67 populations & 1,84 & 87,29 & 2,79 & 1,51 & 1,52 & 96,56 & 2,21 & 11,87 \\
\hline Signification (test $t$ ) & & $* * *$ & & $* * *$ & & & & $* *$ \\
\hline $\begin{array}{l}\text { Albumen blanc (white endosperm) } \\
61 \text { populations }\end{array}$ & 1,83 & 84,71 & 2,82 & 1,55 & 1,53 & 96,13 & 2,18 & 11,91 \\
\hline $\begin{array}{l}\text { Albumen jaune (yellow endos- } \\
\text { perm) } 114 \text { populations }\end{array}$ & 1,83 & 84,88 & 2,68 & 1,66 & 1,50 & 97,03 & 2,18 & 12,11 \\
\hline Signification (test $t$ ) & & & & $* * *$ & & & & \\
\hline
\end{tabular}

Signification (Significance) : ${ }^{* *}$ (seuil 1 p. 100 limit), ${ }^{* * *}$ (seuil 1 p. 100 limit). 
4) l'association fortuite par dérive génétique ou plus simplement par effet d'échantillonnage ;

5) le jeu des 4 premiers facteurs entre chacun des 2 caractères et un $3^{\mathrm{e}}$ caractère.

Plus l'échantillonnage des génotypes sera large, plus l'importance des facteurs $2,3,4$ sera réduite.

Nous examinerons, cas par cas, les 15 associations significatives entre 2 caractères, puis les cas ou aucune relation n'est trouvée entre des caractères que l'on supposait liés :

1) vigueur de départ et santé de l'épi (rVD. SE = $\left.+0,26^{* *}\right)$. Une plus grande vigueur de départ est statistiquement associée à une meilleure santé de l'épi. Cette relation peut être due à un facteur de type 5 , le $3^{\mathrm{e}}$ caractère en jeu étant la qualité de la semence. Les semences des populations étudiées ont en effet été fabriquées dans des conditions climatiques comparables à celles de l'essai à StMartin-de-Hinx. Des différences d'état sanitaire de l'épi pourraient entraîner une mauvaise qualité de semence et une faible vigueur de départ;

2) précocité de floraison femelle et protandrie $\left(\mathrm{rFF}, \mathrm{PA}=+0,48^{* *}\right)$. Une floraison plus précoce est statistiquement associée à une protandrie moins marquée.

Il s'agit ici de 2 caractères de développement. Cette liaison relativement étroite peut être due à un facteur de type 1, la précocité génétique jouant à la fois sur la précocité femelle et sur l'intervalle entre les floraisons des 2 sexes

3) précocité de floraison femelle et pourriture des tiges ( $\mathrm{rFF}$. PT $\left.=-0,28^{* *}\right)$. Une floraison plus précoce est statistiquement associée à une augmentation de la pourriture des tiges.

Les observations de pourriture des tiges ont été réalisées à la maturité apparente de chaque population. Nous n'avons aucune explication satisfaisante pour cette relation;

4) précocité de floraison femelle et verse à la récolte $\left(\mathrm{rFF} . \mathrm{VR}=+0,22^{* *}\right)$. Une floraison plus précoce est statistiquement associée à une diminution de la verse à la récolte. Ici encore nous n'avons pas d'explication satisfaisante ;

5) précocité de floraison femelle et nombre d'épis pour 100 pieds ( $\left.\mathrm{rFF} . \mathrm{NE}=-0,15^{*}\right)$. Une floraison plus précoce est statistiquement associée à une augmentation du nombre d'épis pour 100 pieds. Cette corrélation va à l'encontre de lopinion fréquemment répandue selon laquelle les maïs prolifiques en épis seraient en général plus tardifs. De fait nombre de populations relativement tardives (par exemple populations hongroises) produisent peu d'épis et paraissent manquer d'adaptation à nos conditions culturales ;

6) protandrie et pourriture des tiges (rPA.PT = $\left.-0,19^{*}\right)$. Une protandrie plus marquée est statistiquement associée à une moindre pourriture des tiges. Cette association peut être du type 5 . En effet, un $3^{\mathrm{e}}$ caractère, la précocité, très liée à la protandrie, est en corrélation plus significative avec une diminution de la pourriture des tiges ;

7) protandrie et nombre d'épis pour 100 pieds (rPA. NE $=-0,31^{* *}$ ). Une protandrie plus marquée est statistiquement associée à une diminution du nombre d'épis pour 100 pieds. Ce phénomène du type 1 peut être rattaché à la physiologie des relations puits-source photosynthétique et aux corrélations entre bourgeons (BERTIN et al., 1976) ;

8) pourriture des tiges et nombre d'épis pour 100 pieds (rPT . NE $\left.=+0,15^{*}\right)$. Une pourriture des tiges accentuée accompagne statistiquement un plus grand nombre d'épis pour 100 pieds. Notre expérience de la sélection pour la prolificité en épis confirme malheureusement cette association. On peut proposer une relation de type 1 : une meil- leure prolificité en épis modifierait la relation puits-source photosynthétique en faveur des épis, ce qui sensibiliserait la moelle des tiges qui joue un rôle de stockage intermédiaire dans la translocation des sucres;

9) pourriture des tiges et santé de l'épi (rPT.SE = $\left.-0,42^{* *}\right)$. Une pourriture plus marquée est statistiquement associée à une moins bonne santé de l'épi. Cette relation très nette peut être due à plusieurs facteurs de type 1 :

a) progression directe des champignons pathogènes vers l'épi ;

b) le même mécanisme de résistance influe sur la réaction des 2 organes;

c) la destruction des tissus de la tige pourrait sensibiliser l'épi ;

10) pourriture des tiges et teneur en protéine du grain ( $\mathrm{rPT} . \mathrm{PG}=+0,17^{*}$ ). Une pourriture des tiges accentuée accompagne statistiquement une meilleure teneur du grain en protéine. La même association a été trouvée chez des hybrides tirés de la population «Illinois High Protein » (BOYAT et al., 1979).

Nous ne disposons d'aucune explication satisfaisante pour cette relation;

11) santé de l'épi et teneur en protéine du grain ( $\mathrm{rSE} . \mathrm{PG}=-0,23^{* *}$ ). Une moins bonne santé de l'épi est statistiquement associée à une meilleure teneur du grain en protéine. Nous n'avons pas d'explication satisfaisante. On peut noter que les 3 caractères de pourriture des tiges, de santé de l'épi, de teneur en protéine du grain sont en corrélation les uns avec les autres ;

12) texture cornée du grain et précocité de floraison femelle (tabl. 4). Les populations cornées sont en moyenne plus précoces de près de 4 jours en comparaison avec les dentées. Il est possible que l'échantillonnage joue un rôle car des populations dentées peuvent avoir une composante récente de maïs nord-américain denté plus tardif. En Amérique du Nord, beaucoup de types précoces sont également à grain corné ( Northern flint », « Gaspé ») ;

13) texture cornée du grain et pourriture des tiges (tabl. 4). Les populations cornées ont une note moyenne de pourriture des tiges de 1,69 contre 1,51 pour les populations dentées. Il pourrait s'agir d'un facteur de type 5 mettant en relation un $3^{\mathrm{e}}$ caractère, ici la précocité de floraison femelle qui est associée aux 2 facteurs étudiés ;

14) texture cornée du grain et teneur en protéine du grain (tabl. 4). Les populations cornées ont une teneur en protéine moyenne de 12,15 p. 100 contre 11,87 p. 100 pour les dentées. Cette association n'est pas surprenante : la sélection pour une meilleure richesse en protéine du grain s'accompagne parfois d'une transformation de la texture du grain qui devient plus corné (Anonyme, 1974). De même, la texture du grain des lignées pures est généralement plus cornée que celle des hybrides et leur teneur en protéine est plus élevée ;

15) couleur jaune du grain et pourriture des tiges (tabl. 4). Les populations à grain jaune ont une note moyenne de pourriture des tiges de 1,66 contre 1,55 pour les populations à grain blanc. Nous n'avons aucune explication satisfaisante à fournir pour cette relation.

Enfin, l'indépendance statistique entre certains caractères nous a paru remarquable pour 3 couples :

a) vigueur de départ et précocité de floraison femelle. Les populations précoces ne paraissent donc pas mieux adaptées à nos conditions en début de végétation ;

$b$ ) pourriture des tiges et verse à la récolte. Ces deux caractères ont pourtant été mesurés simultanément ;

c) vigueur de départ et texture du grain. 
L'indépendance entre ces 2 caractères contraste avec l'expérience des sélectionneurs qui utilisent le matériel européen corné (en combinaison avec les maïs dentés nordaméricains) pour améliorer la vigueur de départ et l'adaptation aux conditions fraîches du printemps européen.

\section{CONCLUSION}

Le matériel introduit représente manifestement une variabilité assez large pour tous les caractères étudiés malgré la prépondérance de la texture cornée du grain qui diffère donc de celle des populations dentées du «corn belt $»$ des Etats-Unis.

Pour les 6 caractères d'intérêt économique (vigueur de départ, précocité de floraison femelle, pourriture des tiges, verse à la récolte, santé de l'épi, teneur en protéine du grain), nous avons trouvé 6 associations dont 3 favorables (vigueur de départ et santé de l'épi, précocité de floraison femelle et moindre verse à la récolte, moindre pourriture des tiges et meilleure santé de l'épi), 3 défavorables (précocité de floraison et pourriture des tiges, pourriture des tiges et meilleure teneur en protéine du grain, mauvaise santé de l'épi et meilleure teneur en protéine du grain).
Nous avons également trouvé 4 associations entre les caractères physiologiques (protandrie, nombre d'épis pour 100 pieds) et les caractères d'intérêt économique, 4 associations entre les caractères qualitatifs (texture et couleur du grain) et les caractères d'intérêt économique, une association entre les 2 caractères physiologiques (protandrie et nombre d'épis pour 100 pieds).

Ces associations, souvent difficiles à interpréter, ne sont pas très étroites et devraient en cas de besoin pouvoir être modifiées par la sélection.

L'étude et l'exploitation de ce matériel peuvent être poursuivies en employant différentes méthodes:

- étude de la valeur en combinaison, qui permet d'éliminer les effets de consanguinité ;

- étude de la variabilité intrapopulation;

- regroupements variétaux ;

- sélection récurrente des populations ;

- apports de variabilité extérieure.

Il sera alors nécessaire de vérifier si les associations constatées persistent au sein d'une variabilité améliorée et diversifiée.

Reçu le 8 septembre 1980. Accepté le 17 mars 1982.

\section{RÉFÉRENCES BIBLIOGRAPHIQUES}

Anonyme, 1974. Seventy generations of selection for oil and proteins in Maize. Crop Science Society of America.

Bertin C., Panouillé A., Rautou S., 1976. Obtention de variétés de maïs prolifiques en épis, productives en grain et à large adaptation écologique. Ann. Amélior. Plantes, 26 (3), 387-418.

Boyat A., Kaan F., Panouillé A., Rautou S., 1979. Amélioration du maïs pour la teneur en protéines du grain. Symposium I.A.E.A. F.A.O. ; Seed Protein improvement in cereals and grain legumes, Neuherberg, 4-8 septembre 1978, 2, 151-164.

Brandolini A., 1970. Razze europee di maïs. Maydica, 15, 5-27.

Edwards R. J., Leng E. R., 1965. Classification of some indigenous maize collections from southern and south eastern Europe. Euphytica, 14, 2, 161-169.

Monteagudo A., 1967. Phylogenetic trees of the spanish maize races. Proc. 4th meeting of the Maize and Sorghum section of Eucarpia. Montpellier, 116-123.
Muresan T., Cosmin O., 1967. Valeur des mais roumains du sud du pays comme source de matériel de départ pour les travaux d'amélioration. Proc. 4th meeting of the Maize and Sorghum section of Eucarpia. Montpellier, 77-102.

Pavlicic J., 1969. Contribution to a preliminary classification of European open pollinated maize varieties. Proc. 5th Meeting of the Maize and Sorghum section of Eucarpia, 93-107.

Pavlicic J., Trifunovic V., 1967. A study of some important ecologic corn types in Yugoslavia and their classification. J. Sci. Agric. Res., 19, 44-62.

Sanchez-Monge E. P., 1962. Razas de Maiz en Espana. Min. de Agric., Madrid monografias, $\mathrm{n}^{\circ} 13$.

Sanchez-Monge E. P., 1964. Races of maize in Spain. 3rd meeting of the maize and sorghum section of Eucarpia, Vienna, 112-113. 